Research Article

\title{
The Synthesis and Application of Nitrogen-Doped Graphene Quantum Dots on Brilliant Blue Detection
}

\author{
Li Jin $\left(\mathbb{D},{ }^{1}\right.$ Ying Wang, ${ }^{1}$ Fengkai Yan, ${ }^{2}$ Jianpo Zhang $\left(\mathbb{D},{ }^{1}\right.$ and Fangli Zhong $\mathbb{D}^{1}$ \\ ${ }^{1}$ School of Chemical and Pharmaceutical Engineering, Jilin Institute of Chemical Technology, Jilin 132022, China \\ ${ }^{2}$ Production Department, Chemical Fertilizer Plant of Jilin Petrochemical Corporation, PetroChina, Jilin, Jilin 132021, China \\ Correspondence should be addressed to Fangli Zhong; zhongfl@jlict.edu.cn
}

Received 13 December 2018; Revised 27 February 2019; Accepted 18 March 2019; Published 7 May 2019

Academic Editor: Hassan Karimi-Maleh

Copyright (C) $2019 \mathrm{Li}$ Jin et al. This is an open access article distributed under the Creative Commons Attribution License, which permits unrestricted use, distribution, and reproduction in any medium, provided the original work is properly cited.

\begin{abstract}
Nitrogen-doped graphene quantum dots had been successfully synthesized and characterized by using transmission electron microscope, X-ray photoelectron spectroscopy, absorbance spectrum, fluorescence emission spectrum, and fluorescence decay curve. TEM results indicated that the diameters of the as-prepared nitrogen-doped graphene quantum dots were in the range of $2-5 \mathrm{~nm}$ and the lattice space is about $0.276 \mathrm{~nm}$; Raman spectrum result indicated that there were two characteristic peaks, generally named D $\left(\sim 1408 \mathrm{~cm}^{-1}\right)$ and $\mathrm{G}\left(\sim 1640 \mathrm{~cm}^{-1}\right)$ bands; both TEM and Raman spectrum results indicated that the assynthesized product was graphene quantum dots. Deconvoluted high resolution XPS spectra for C1s, O1s, and N1s results indicated that there are $-\mathrm{NH}-,-\mathrm{COOH}$, and $-\mathrm{OH}$ groups on the surface of nitrogen-doped graphene quantum dot. Fluorescence emission spectrum indicated that the maximum fluorescence emission spectrum of nitrogen-doped graphene quantum dots was blue shift about $30.1 \mathrm{~nm}$ and the average fluorescence decay time of nitrogen-doped graphene quantum dots increased about $2 \mathrm{~ns}$, compared with graphene quantum dots without doping of nitrogen. Then, the as-prepared nitrogen-doped graphene quantum dots were used to quantitatively analyze brilliant blue based on the fluorescent quenching of graphene quantum dots, and the effect of $\mathrm{pH}$ and reaction time on this fluorescent quenching system was also obtained. Under selected condition, the linear regression equations were $F_{0} / F=0.0087$ (brilliant blue) +0.9553 and $F_{0} / F=0.01205$ (brilliant blue) +0.6695 , and low detection limit was $3.776 \mu \mathrm{mol} / \mathrm{L}(3.776 \mathrm{nmol} / \mathrm{mL})$. Once more diluted N-GQDs $(0.05 \mathrm{mg} / \mathrm{mL})$ were used, the low detection limit could reach $94.87 \mathrm{nmol} / \mathrm{L}$. Then, temperature-dependent experiment, absorbance spectra, and dynamic fluorescence quenching rate constant were used to study the quenching mechanism; all results indicated that this quenching process was a static quenching process based on the formation of complex between nitrogen-doped graphene quantum dots and brilliant blue through hydrogen bond. Particularly, this method was used to quantitatively analyze the wine sample, of which results have a high consistence with the results of the spectrophotometric method; demonstrating this fluorescence quenching method could be used in practical sample application.
\end{abstract}

\section{Introduction}

Graphene quantum dots (GQDs) have been extensively used in drug delivery, sensor, bioimaging, photocatalysis, delectrocatalysis, and optoelectronic devices [1] due to their superior properties. Particularly, GQDs used as a fluorescent sensor based on the quenching of its fluorescence emission are becoming a hot topic [2-9]. Different synthesis methods have been investigated to get graphene quantum dots such as a novel hydrothermal method [10], chemical oxidation and exfoliation method [11], and citric acid splitting method
$[12,13]$. However, most of these reported GQDs are not satisfactory owing to low fluorescence emission intensity and complicated preparation procedures.

Luminescent properties of GQDs are depending on many factors such as size, shape, surface oxidation degree, and surface functionalization. Nowadays, the synthesis of heteroatom- (boron [14], sulfur [15], fluorine [16], phosphorus [17], and nitrogen [18]) doped GQDs has attracted increasing attention. Experimental results demonstrate that nitrogen is a good candidate for chemical doping of GQDs, because nitrogen with five valence electrons could react with 
graphene atoms to form strong valence bonds. However, most of these methods are not satisfactory owing to expensive equipment required, severe preparation conditions, and complicated preparation procedures [19]. Compared to electrochemical method, hydrothermal method may be a good candidate owing to its easy operation and relative inexpensive equipment [20]. Guo et al. [21] reported a novel onepot hydrothermal method to synthesize the GQDs with bifunctional groups $\left(-\mathrm{OH}\right.$ and $-\mathrm{NH}_{2}$ ) using a polycyclic aromatic hydrocarbon as the precursor, but heating temperature is a little high and the use of organic solvents is not green. Consequently, a more easy and fast synthesis method should be investigated.

Brilliant blue (BB), a kind of synthetic colorant, has been widely used in food additive [22]. But research results show that excessive additive of brilliant blue will affect the quality and safety of food products and the maximum quantity added in food is no more than $25 \mathrm{mg} / \mathrm{kg}$ in China. Hence, accurate and reliable determination methods for a quantitative analysis of brilliant blue have been explored. Among these methods, high performance liquid chromatography (HPLC) $[23,24]$ has advantages of better resolution and fast, but needs complicated sample pretreatment process, but not suitable to analyze a large sum of samples. Spectrophotometry $[25,26]$ is simple and fit for analyzing batch samples, but needs preconcentration technique. Electrochemiluminescence (ECL) [27] is also a sensor for brilliant blue analysis which has good chemical stability, wide $\mathrm{pH}$ range, high luminous efficiency, and excellent electrochemical performance, but the surface treatment of electrode is a little time-consuming and complicated. Other methods such as capillary electrophoresis [28] and voltammetry [29, 30] have also been developed to analyze brilliant blue; however, some disadvantages, such as relatively costly instruments, skilled operator, complicated sample pretreatment process, and time-consuming, seriously limit its scope of application and fields. Thus, researchers have to make great efforts in developing other sensitive brilliant blue analysis methods. Quantum dots with fluorescence properties may be a good ?candidate, of which decreasing of fluorescence intensity has a good linear relationship with the concentration of analyte. Nitrogen-doped graphene quantum dots would be a suitable candidate for a quantitative analysis of brilliant blue. Also, from what I could gather, the interaction between brilliant blue and nitrogen-doped graphene is relatively unexplored.

In this paper, a simple and fast method was investigated to synthesize N-GQDs based on directly heating citric acid and urea on an electric stove for $5 \mathrm{~min}$, and then N-GQDs were used as a fluorescent probe for a quantitative analysis of brilliant blue based on the fluorescence quenching of $\mathrm{N}$ GQDs. This quenching method with the advantage of simple, fast, and inexpensive should have significant implications to guarantee the safety of foods and the rights of consumers.

\section{Material and Method}

2.1. Material and Instrumentation. Brilliant blue was purchased from Shanghai Maikun Co., Ltd. Citric acid and urea were obtained from Sinopharm Chemical Reagent Co., Ltd. (China). Dialysis bags (molecular weight cut-off: $1000 \mathrm{Da}$ ) were bought from Sangon Biological Technology \& Services Co., Ltd. (China). The reagents used in the experiment are all analytically pure. Doubly distilled water was used throughout this experiment.

F-280 spectrofluorophotometer, which adopts a pulsed xenon lamp as an excitation light source, was used to get fluorescence spectra. Shimadzu UV-2550 UV-visible spectrometer was used to study the ultraviolet-visible spectroscopy. The FT-IR spectrum was carried out by the $\mathrm{KBr}$ method on a NICOLET6700 spectrometer. The XPS spectra were recorded with a Thermo Scientific K-Alpha. The Raman spectrum was recorded with a Raman detection system, including a He-Ne laser $(20 \mathrm{~mW}, 633 \mathrm{~nm}$, Uniphase), a set of Raman filters (Semrock), and a spectrometer (IHR 320, JY, France).

2.2. Preparation of $N-G Q D s$. The synthesis method of $\mathrm{N}-$ GQDs is close to the results reported in some literature $[12,13]$. Briefly, $0.2 \mathrm{~g}$ citric acid and $0.2 \mathrm{~g}$ urea in a $50 \mathrm{~mL}$ beaker were heated directly on the electric stove for about $5 \mathrm{~min}$; after the citric acid changes to an orange liquid, $5 \mathrm{~mL}$ of ?distilled water was added and sonicated for about $2 \mathrm{~min}$, then adjusted to $\mathrm{pH} 7.0$ by $\mathrm{NaOH}$ solution, and then dialyzed overnight. The as-prepared $\mathrm{N}$ GQDs are concentrated by rotary evaporator and dried at $80^{\circ} \mathrm{C}$. After weighing, N-GQD solution of $5 \mathrm{mg} / \mathrm{mL}$ was obtained and stored in a refrigerator at 4 centigrade.

2.3. The Quantitative Analysis of Brilliant Blue with N-GQDs. $10 \mu \mathrm{L}$ of brilliant blue sample solution was added into $0.1 \mathrm{~mL}$ of N-GQD solution and diluted to $2 \mathrm{~mL}$ with distilled water ( $\mathrm{pH}=7$, the concentration of $\mathrm{N}-\mathrm{GQDs}$ is $0.5 \mathrm{mg} / \mathrm{mL}$ ). After this reaction mixture was incubated for $5 \mathrm{~min}$ at room temperature, fluorescence emission spectrum solution was obtained, the exciting wavelength is $350 \mathrm{~nm}$, and excitation and emission monochromator slit width of fluorescence spectrophotometer were both $5.0 \mathrm{~nm}$.

\section{Results and Discussion}

3.1. Characterization of Nitrogen-Doped Graphene Quantum Dots. The as-synthesized N-GQD product was characterized by transmission electron microscopy (TEM) images, Raman spectrum, and XPS spectrum. Figure 1(a) shows that NGQDs were highly crystalline with a mean particle diameter of $2-5 \mathrm{~nm}$, and the lattice space (about $0.276 \mathrm{~nm}$ ) is a little longer than that of GQDs due to doping of nitrogen [7]. The diameter distribution $(d=3 \pm 0.14 \mathrm{~nm})$ was determined from $n=18$ particles, as shown in Figure 1(b). The Raman spectrum of graphene quantum dots (Figure S1) shows that there were two characteristic peaks, generally named D $\left(\sim 1408 \mathrm{~cm}^{-1}\right)$ and $\mathrm{G}\left(\sim 1640 \mathrm{~cm}^{-1}\right)$ bands; both TEM and Raman spectrum results indicated that the as-synthesized product was graphene quantum dots. The AFM characterization is shown in Figure S2, which indicate that very little aggregation is present. 


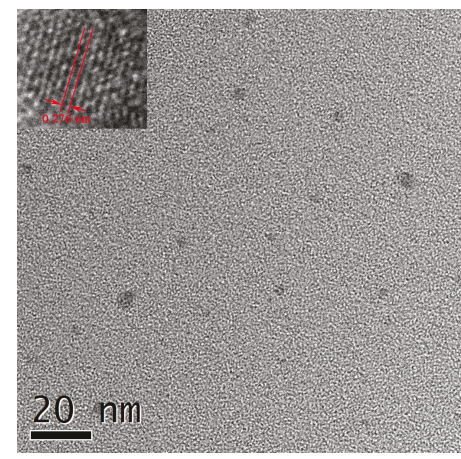

(a)

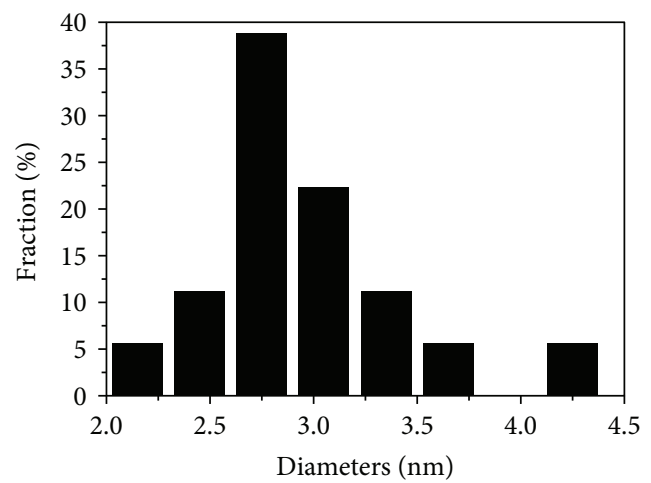

(b)

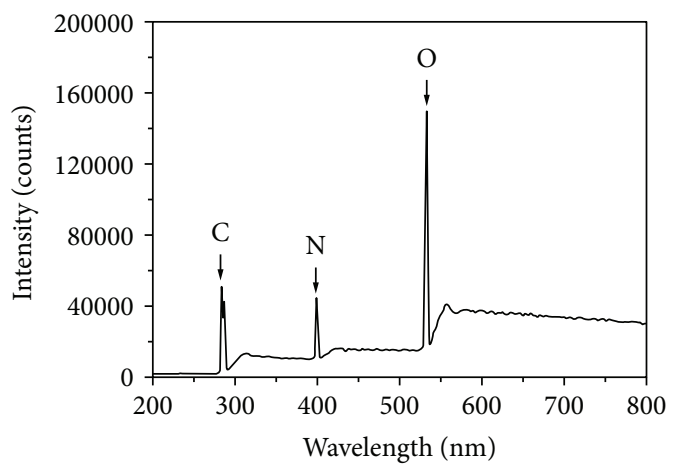

(c)

FIgURE 1: TEM images (a), the diameter distribution as manually estimated from $n=18$ particles is shown $(d=3 \pm 0.14 \mathrm{~nm})(\mathrm{b})$ and XPS spectra (c) of N-GQDs.

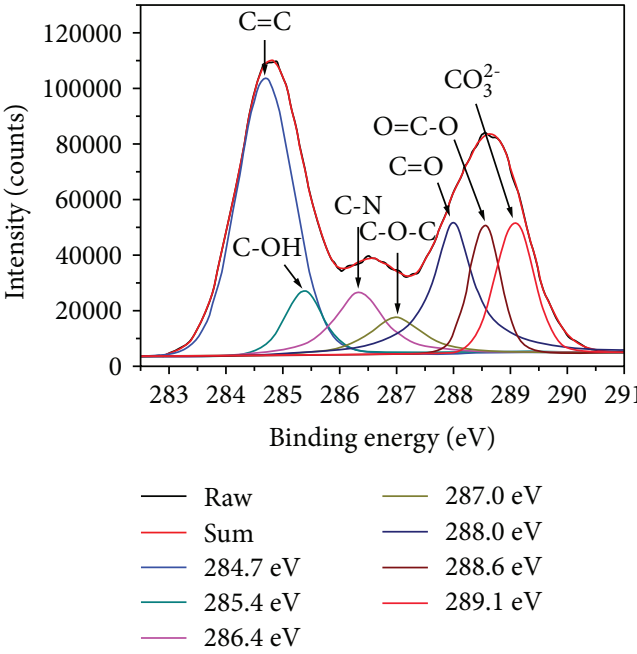

(a)

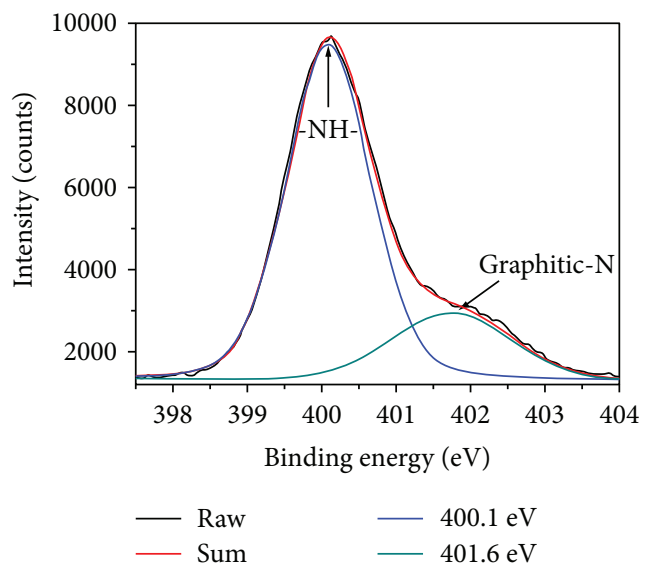

(b)

FIGURE 2: The C1s (a) and N1s (b) spectra of N-GQDs studied by means of XPS-peak-differentiation-imitating analysis.

Elements in N-GQDs were analyzed by XPS spectrum. As shown in Figure 1(c), there are three characteristic peaks, which are corresponding to $\mathrm{C} 1 \mathrm{~s}, \mathrm{O} 1 \mathrm{~s}$, and $\mathrm{N} 1 \mathrm{~s}$, and the $\mathrm{C}, \mathrm{O}$, and $\mathrm{N}$ atomic ratio is $3.6: 2.2: 1$. Deconvoluted high resolution XPS spectra for C1s, O1s, and N1s were also studied. As shown in Figure 2(a), seven fitting peaks at 284.7, 285.4,
286.4, 287.0, 288.0, 288.6, and $289.1 \mathrm{eV}$ are corresponded to the characteristic peaks of $\mathrm{C}=\mathrm{C}$ (graphitic graphene), C$\mathrm{OH}, \mathrm{C}-\mathrm{N}, \mathrm{C}-\mathrm{O}-\mathrm{C}, \mathrm{C}=\mathrm{O}, \mathrm{O}=\mathrm{C}-\mathrm{O}$, and $\mathrm{CO}_{3}{ }^{2-}$. The peak at $286.4 \mathrm{eV}$, assigned to the $\mathrm{C}-\mathrm{N}$, indicates that $\mathrm{N}$ is covalently bonded to C. As shown in Figure 2(b), the peak at 400.1 is assigned to the $-\mathrm{NH}$ - and the peak at $401.6 \mathrm{eV}$ is assigned 


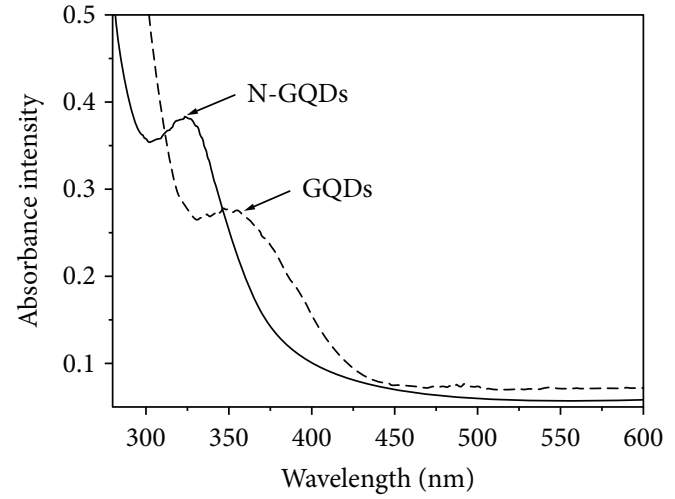

(a)

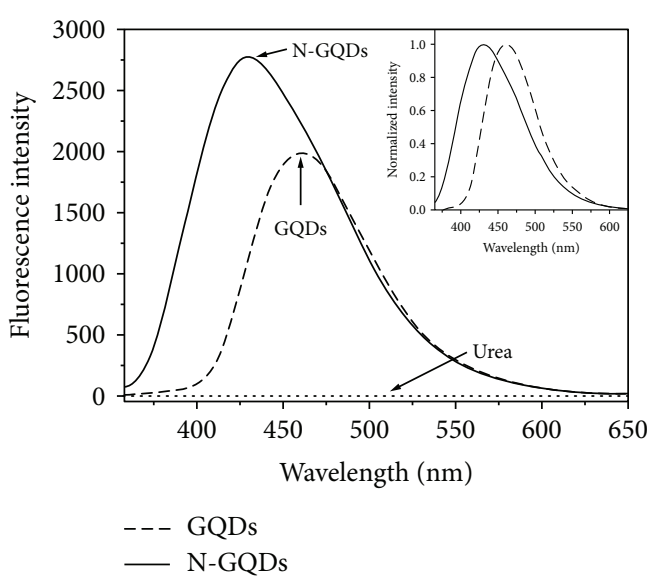

(b)

FIGURE 3: Absorbance spectra (a) and fluorescence emission spectra (b) of GQDs and N-GQDs.

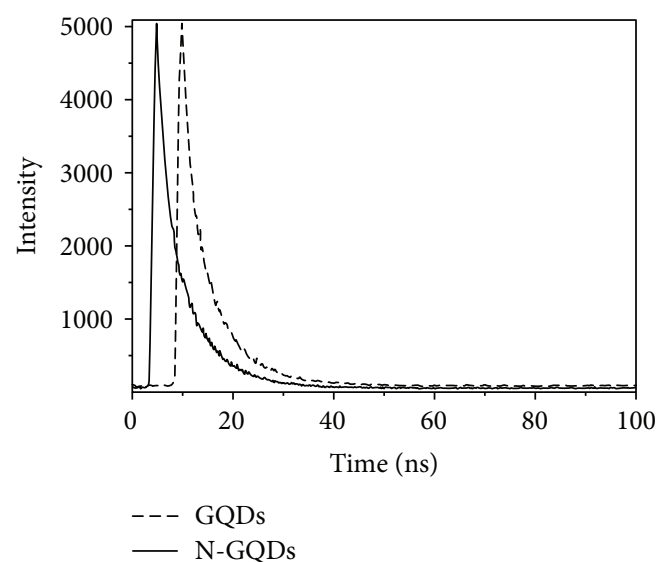

FIGURE 4: Fluorescence decay curves of N-GQDs (solid line) and GQDs (dash line).

to the graphitic $\mathrm{N}$ subpeak [31]. XPS results indicated that there are $-\mathrm{NH}-,-\mathrm{COOH}$, and - $\mathrm{OH}$ groups on the surface of nitrogen-doped graphene quantum dot.

The luminescence property of the as-prepared N-GQDs was also characterized by the absorbance and fluorescence spectra. Compared with that of GQDs (synthesized in the same condition as a controlled experiment), the maximum absorbance wavelength of N-GQDs has blue shifted about $29.1 \mathrm{~nm}$ (Figure 3(a)). And similar results could be obtained from the maximum fluorescence emission spectrum (Figure 3(b)); there is a blue shift (about $30.1 \mathrm{~nm}$ ) compared with that of GQDs (there has hardly any fluorescence emission synthesized by urea in the same condition as a controlled experiment). These blue shifts may be due to the decreasing of diameter of the as-prepared N-GQDs.

Furthermore, the fluorescence decay curves of both GQDs and N-GQDs were shown in Figure 4. The emission kinetics of both GQDs and N-GQDs fitted multiexponentially with time constants, respectively. The average fluorescence decay time of GQDs $\left(\tau_{0}=6.14 \mathrm{~ns}\right)$ and N-GQDs $\left(\tau_{0}=8.1 \mathrm{~ns}\right)$ was calculated by the weighted average method

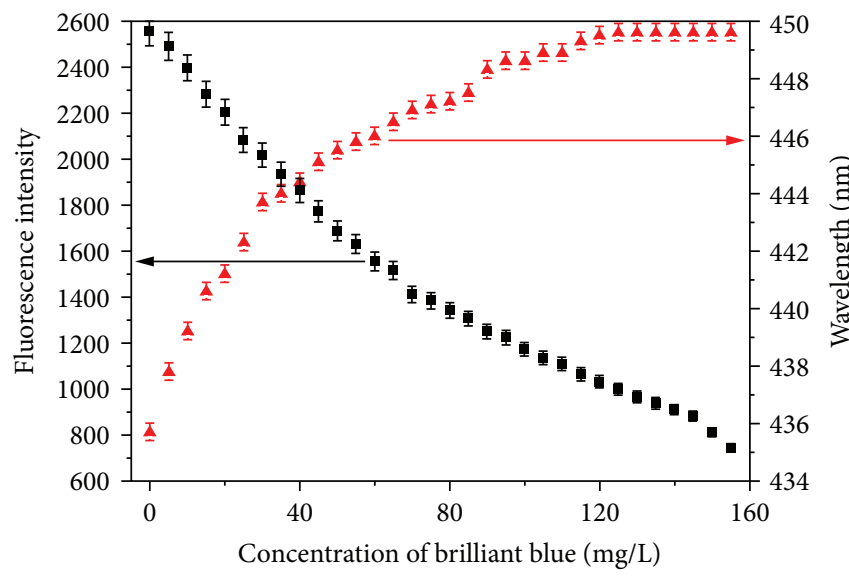

FIgURE 5: Brilliant blue concentration-dependent fluorescence emission of N-GQDs (the concentration of N-GQDs was $0.5 \mathrm{mg} / \mathrm{mL}$, and $\lambda_{\mathrm{ex}}=350 \mathrm{~nm}$ ).

[32]. Since multiexponential dynamics arise from surface traps, surface traps of N-GQDs decreases and the redox energy level shifts to lower energies, compared to that of QGDs, and the average fluorescence decay time of N-GQDs increases. This may be the reason why the fluorescence intensity of N-GQDs increased compared with GQDs. All these results indicated that nitrogen has been successfully doped in the GQDs and there are carboxyl and hydroxyl groups on the surface of N-GQDs. Above all, N-GQDs were successfully synthesized.

3.2. The Interaction between Brilliant Blue and N-GQDs. Interestingly, when brilliant blue was adopted into N-GQD solution, the fluorescence emission intensity and peak position of N-GQDs changed significantly, which were illustrated in Figure 5 (the original fluorescent spectra are illustrated in supporting information Figure S3 in ESI $\dagger$ ). The fluorescence emission intensity of N-GQDs will decrease $69.64 \%$, when $0.155 \mathrm{~g} / \mathrm{L}$ of brilliant blue was added. While the maximum fluorescence emission wavelength of N-GQDs redshift 


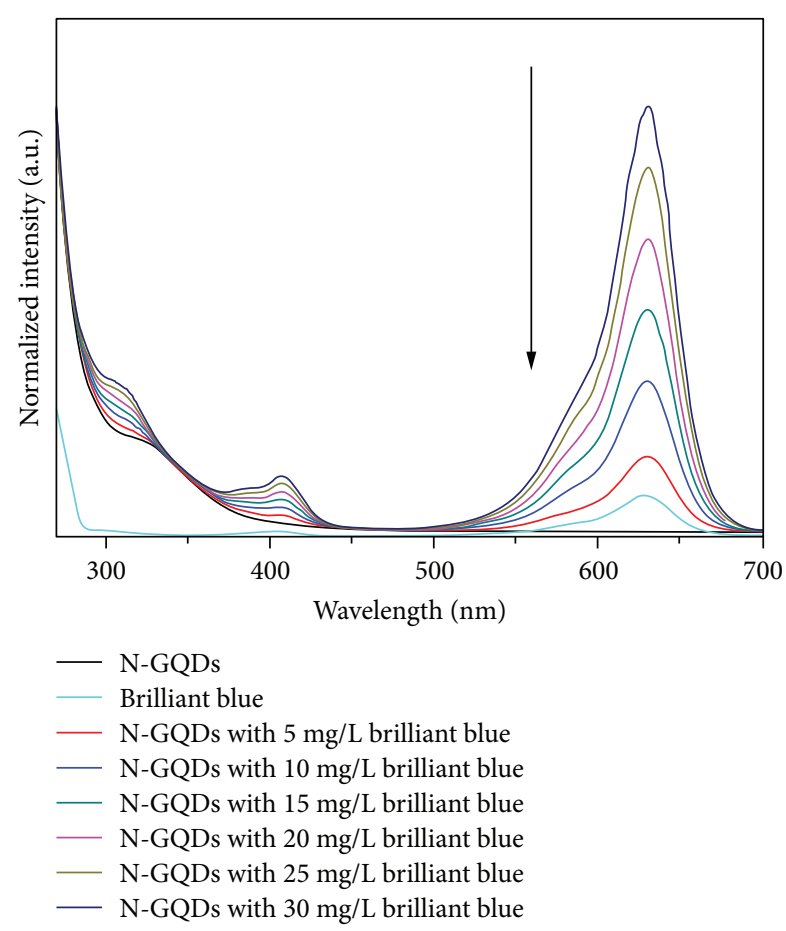

FIgURE 6: Absorbance spectra of N-GQDs with different concentration of brilliant blue.

rapidly initially and then gets constant gradually after the concentration of brilliant blue is more than $120 \mathrm{mg} / \mathrm{L}$, and the maximum redshift of the peak position is $13.9 \mathrm{~nm}$. The mechanism is similar to that we have reported [33]. Brilliant blue is a kind of synthetic colorant with two $-\mathrm{SO}^{3-}$ group, while N-GQDs have carboxyl groups on its surface, so N-GQDs could react with brilliant blue through hydrogen bond.

In order to further elucidate the quenching mechanism of $\mathrm{N}$-GQDs, absorbance spectra and temperature-dependent experiment were introduced. As shown in Figure 6, there is an evident blue shift in the UV-visible absorption spectra of N-GQDs, which indicates that a new complex between brilliant blue and N-GQDs was formed.

This may be because there are carboxyl groups on the surface of graphene quantum dots and $-\mathrm{SO}^{3-}$ groups on the surface of brilliant blue (as shown in Figure S4 in ESI $\dagger$ ), and there has a strong electronegativity between the hydroxyl of the carboxyl group and the sulfonic group; a complex of graphene quantum dots with brilliant blue is formed through hydrogen bond.

Furthermore, the influencing of temperature on this quenching system was conducted, and the equation $F_{0} / F=$ $K_{\mathrm{b}}[\mathrm{C}]+\mathrm{b}$ was applied to handle with the fluorescence data. Figure 7 shows that the slopes of these fitting lines decrease with increasing temperature. As we stated above, a complex of brilliant blue and N-GQDs was formed through hydrogen bond; the stability of the complex would decrease with increasing temperature, so the slope of fitting line decreased.

Then formula $K_{\mathrm{q}}=K_{\mathrm{LB}} / \tau_{0}$ was used to calculate the dynamic fluorescence quenching rate constant $\left(K_{\mathrm{q}}\right)$, where

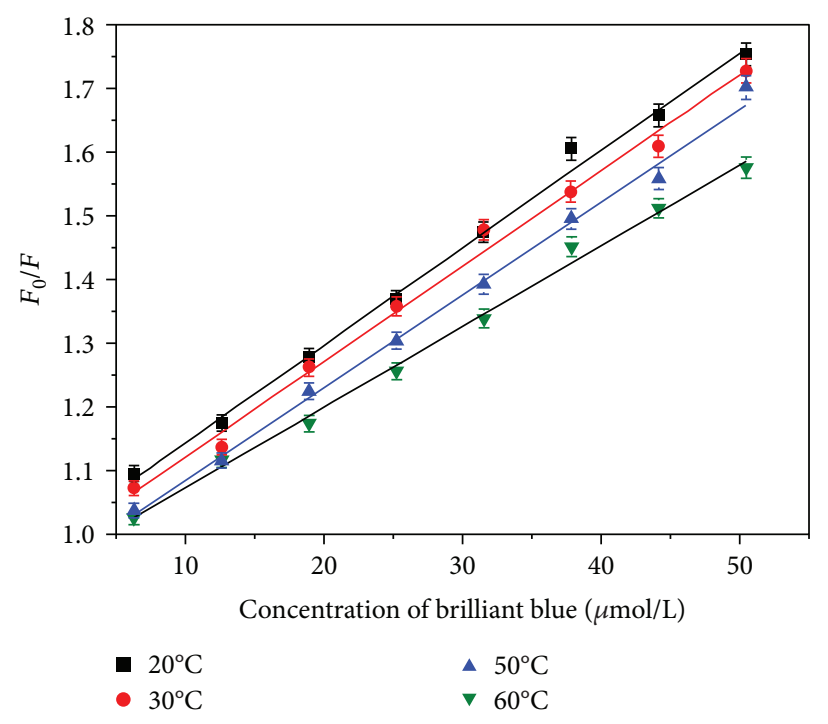

Figure 7: Temperature-dependent response of $F_{0} / F$ to concentrations of brilliant blue.

$\tau_{0}$ represents the average lifetime of fluorescence molecule without quencher. As mentioned above, $\tau_{0}$ is $8.1 \mathrm{~ns}$, so $K_{\mathrm{q}}$ $\left(1.9 \times 10^{12} \mathrm{~mol} \cdot \mathrm{L}^{-1} \cdot \mathrm{s}^{-1}\right)$ could be calculated based on the data of $20^{\circ} \mathrm{C}$, which is far greater than the maximum dynamic quenching constant $\left(2 \times 10^{10} \mathrm{~mol} \cdot \mathrm{L}^{-1} \cdot \mathrm{s}^{-1}\right)$.

Above all, based on the results of UV-visible absorption spectra, the slope of fitting lines from Figure 7, and fluorescence quenching rate constant, we could conclude that this fluorescence quenching progress between N-GQDs and brilliant blue is a static quenching process.

3.3. The Quantitative Analysis of Brilliant Blue. To promote the practical application of this method, the effects of incubation time and $\mathrm{pH}$ on this fluorescence quenching progress were explored and optimized (Figure 8). Both $\mathrm{pH}$ and incubation time have little effect on the $F_{0} / F$ of this sensor system, but in order to get a consistent result, $\mathrm{pH}=7$ and $5 \mathrm{~min}$ incubation time were selected. Furthermore, fluorescence emission and excitation spectra were shown in Figure S5 in ESI†; fluorescence excitation wavelength of $350 \mathrm{~nm}$ and room temperature were selected for further application.

Under selected conditions mentioned above, different concentrations of brilliant blue $(6.306-182.9 \mu \mathrm{mol} / \mathrm{L})$ were added into N-GQD solution. As shown in Figure 9, the linear regression equations between the $F_{0} / F$ and the concentration of brilliant blue $\left(F_{0}\right.$ and $F$ were the fluorescence emission intensity of the N-GQDs with and without brilliant blue, respectively) is $F_{0} / F=0.0087$ (brilliant blue) +0.9553 and $F_{0} / F=0.01205$ (brilliant blue) +0.6695 , with the correlation coefficient $R^{2}$ (0.9964 and 0.9961 , respectively) (the original fluorescent spectra are illustrated in supporting information Figure S3 in ESI $\dagger$ ). Low detection limit (LOD) of $3.776 \mu \mathrm{mol} / \mathrm{L}(3.776 \mathrm{nmol} / \mathrm{mL})$ could be calculated by the equation [32] $\mathrm{LOD}=(3.3 \sigma / k)$, where $\sigma$ stands for the standard deviation of the $y$-intercepts of the regression lines and $k$ stands for the slope of the calibration graph. 


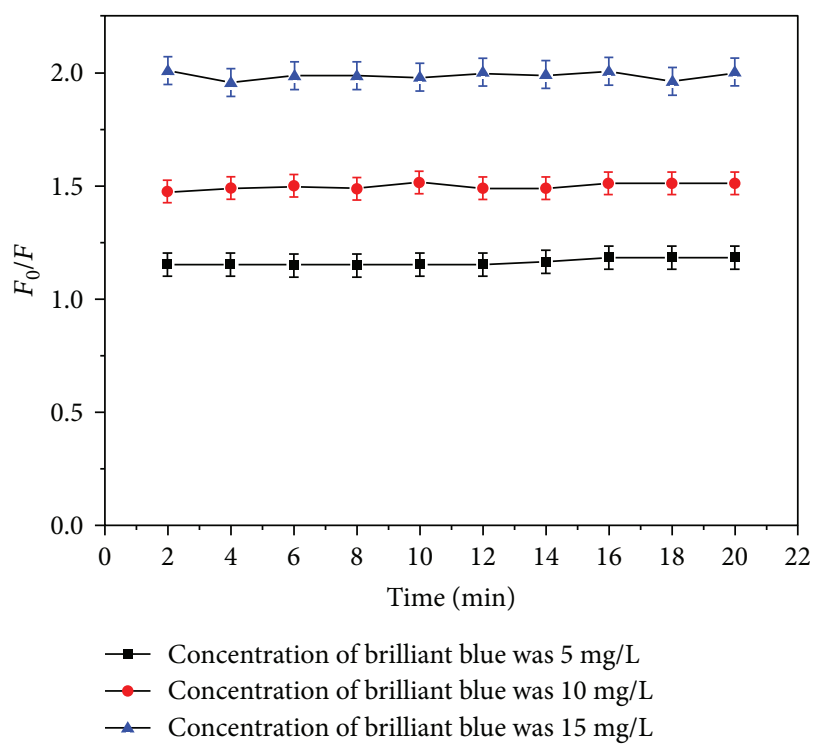

(a)

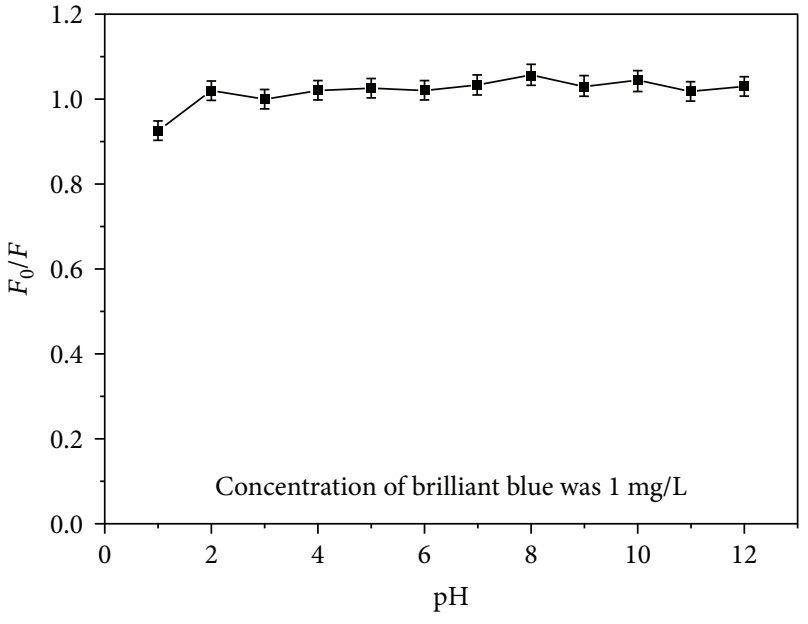

(b)

Figure 8: Effects of $\mathrm{pH}$ and incubation time on the $F_{0} / F$ of this sensor system (the concentration of N-GQDs was $0.05 \mathrm{~g} / \mathrm{L}$ ).

Once more diluted N-GQDs $(0.05 \mathrm{mg} / \mathrm{mL})$ were used, the LOD for brilliant blue could reach $94.87 \mathrm{nmol} / \mathrm{L}$ (as shown in Figure S6 in ESI $\dagger$ ). Although the line range of this method presented was narrow $(0.63-5.05 \mu \mathrm{mol} / \mathrm{L})$, the line range could be adjusted by using different concentrations of $\mathrm{N}$ GQDs to meet the need of different detections in use. Relative standard deviation (equal to $2.9 \%$ ) could be calculated through five replicate detection of $37.84 \mu \mathrm{mol} / \mathrm{L}$ brilliant blue, which indicated this method exhibits good reproducibility.

3.4. The Detection of Wine Sample. The influence of potential components of wine (ethanol, maltose, sucrose, glucose, citric acid, and sodium citrate) and metal ion on this quantitative analysis system was also studied. As shown in Figure 10, except $\mathrm{Cu}^{2+}, \mathrm{Ni}^{2+}, \mathrm{Fe}^{2+}$, and $\mathrm{Fe}^{3+}$, other substances have little effect on the fluorescence emission intensity of $\mathrm{N}$-GQDs. But the concentrations of these substances $\left(\mathrm{Cu}^{2+}, \mathrm{Ni}^{2+}, \mathrm{Fe}^{2+}\right.$, and $\left.\mathrm{Fe}^{3+}\right)$ in real sample were too low to cause disturbance. It demonstrates that this quantitative analysis system has a high selectivity for brilliant blue.

A standard addition method was applied to analyze the concentration of brilliant blue in the wine samples. First of all, $10 \mathrm{~mL}$ of wine samples was diluted to $100 \mathrm{~mL}$ with doubly distilled water (sample test, $\mathrm{pH}=7$ ). The wine analysis samples were obtained by adding a fixed amount of brilliant blue standard solution into the sample test, and the fluorescence emission intensity N-GQDs with the sample test solution was obtained by using the method mentioned above. Fluorescence emission intensity of N-GQDs decreased significantly after wine test solution was added into N-GQD solution. Base on the fluorescence emission intensity of N-GQDs and the linear regression equations, we found that brilliant blue contained in the wine samples was $0.01865 \mathrm{~g} / \mathrm{kg}$. In order to validate the presence method, the brilliant blue detections by the spectrophotometric method (standard working curves

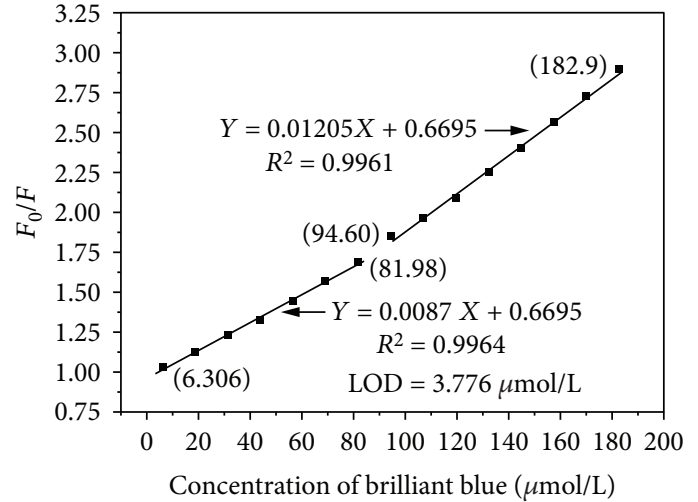

FIgURE 9: Brilliant blue concentration-dependent fluorescence emission of N-GQDs (the concentration of N-GQDs was $0.5 \mathrm{mg} / \mathrm{mL}$, and $\lambda_{\mathrm{ex}}=350 \mathrm{~nm}$ ).

of the spectrophotometric method for a quantitative analysis of brilliant blue were shown in Figure S7) were also performed for these real samples. The concentration of brilliant blue in water and wine detected by both methods was listed in Table 1. The results from the two methods were in high consistence; demonstrating this fluorescence quenching method could be applied for a quantitative analysis of brilliant blue in real samples.

\section{Conclusion}

Nitrogen-doped graphene quantum dots have been successfully prepared and characterized, which was also used as a fluorescence quenching material for a quantitative analysis of brilliant blue in solution, of which line range could be adjusted by using different concentrations of N-GQDs to meet the need of different detections in use. The low detection limit could reach $94.87 \mathrm{nmol} / \mathrm{L}$ by using diluted $\mathrm{N}$ - 


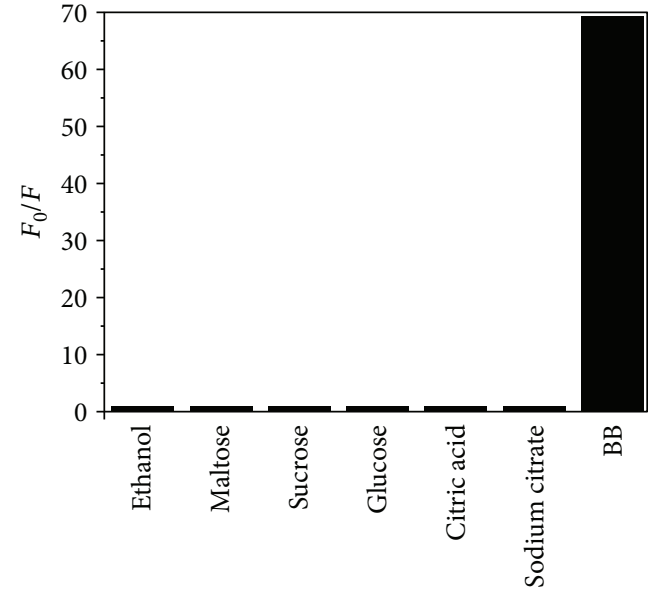

Interfering substance

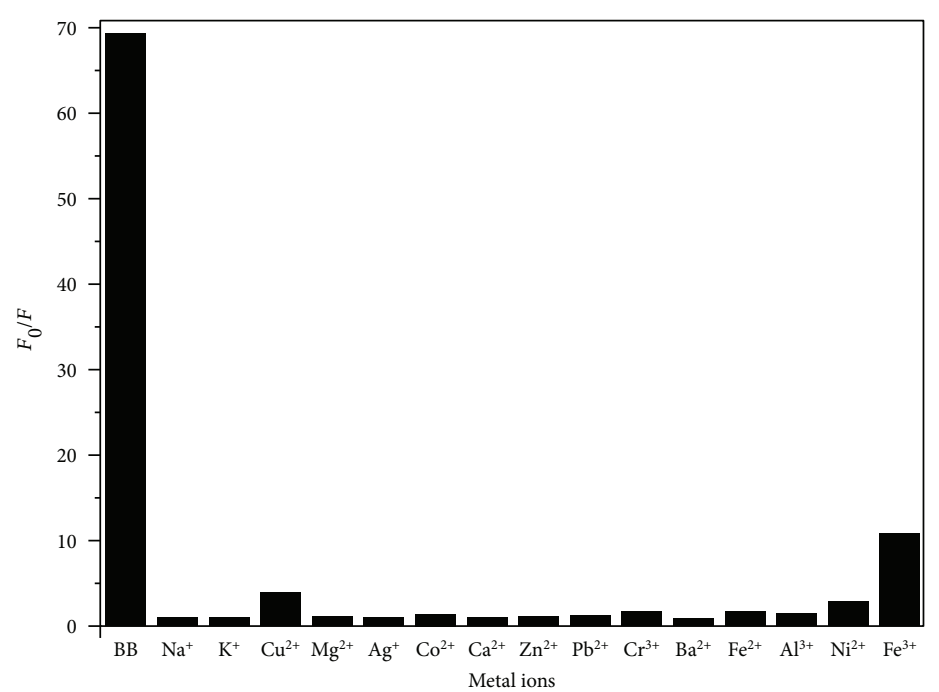

(b)

FIGURE 10: Effects of metal ions on the $F_{0} / F$ of this sensor system under the optimum conditions (the concentration of N-GQDs was $0.05 \mathrm{mg} / \mathrm{mL}$, and $\left.\lambda_{\text {ex }}=350 \mathrm{~nm}\right)$.

TABLE 1: Detection of brilliant blue in water and wine.

\begin{tabular}{lccc}
\hline Samples & $\begin{array}{c}\text { Betected by fluorescence } \\
(\mu \mathrm{mol} / \mathrm{L})\end{array}$ & $\begin{array}{c}\text { Brilliant blue } \\
\text { Detected by spectrophotometric } \\
\text { method }(\mu \mathrm{mol} / \mathrm{L})\end{array}$ & $\begin{array}{c}\text { Consistence } \\
\text { Distilled water }\end{array}$ \\
& 18.92 & 58.53 & $96.88 \%$ \\
& 56.76 & 84.56 & $96.13 \%$ \\
\hline \multirow{3}{*}{ Wine } & 81.98 & 11.03 & $96.46 \%$ \\
& 10.64 & 19.38 & $96.90 \%$ \\
& 18.78 & 33.38 & $97.06 \%$ \\
$97.16 \%$ \\
\hline
\end{tabular}

GQDs $(0.05 \mathrm{mg} / \mathrm{mL})$. Results of absorbance spectra, temperature-dependent experiment, and calculation indicate that it is a static quenching progress. Furthermore, this method was used to analyze brilliant blue in wine samples. Compared with previous approaches for wine detection, this method was more suitable for the future application, as it is a simple, fast, cost-effective, and especially sensitive method for rapidly analyzing wine solution.

\section{Data Availability}

All data included in this study are available upon request by contact with the corresponding author.

\section{Conflicts of Interest}

The authors declare that they have no conflicts of interest.

\section{Acknowledgments}

We gratefully thank the National Natural Science Foundation of China (21405058).

\section{Supplementary Materials}

Brilliant blue concentration-dependent fluorescence emission of N-GQDs, Raman spectrum of the as-synthesized NGQDs, schematic illustration of interaction between $\mathrm{N}$ GQDs and brilliant blue, excitation and emission spectrum of N-GQDs, the absorbance spectra of different concentrations of brilliant blue, brilliant blue concentrationdependent fluorescence emission of N-GQDs, and corresponding standard working curves of the spectrophotometric method for a quantitative analysis of brilliant blue were shown in Figures S1-S6. Figure S1: Raman spectrum of the as-synthesized N-GQDs. Figure S2: AFM image of GQDs (a) and their corresponding height profile (b). Figure S3: ?brilliant blue concentration-dependent fluorescence ?emission of N-GQDs (the concentration of N-CQDs was $0.5 \mathrm{mg} / \mathrm{mL}$, and $\lambda_{\mathrm{ex}}=350 \mathrm{~nm}$ ). Figure S4: schematic illustration of interaction between N-GQDs and brilliant blue. Figure S5: excitation and emission spectrum of N-GQDs. Figure S6: brilliant blue concentration-dependent fluorescence emission of N-GQDs (the concentration of N-GQDs was $0.05 \mathrm{mg} / \mathrm{mL}$, and $\lambda_{\mathrm{ex}}=350 \mathrm{~nm}$ ). Figure $\mathrm{S} 7$ : the 
absorbance spectra of different concentrations of brilliant blue and corresponding standard working curves of the spectrophotometric method for a quantitative analysis of brilliant blue. (Supplementary Materials)

\section{References}

[1] H. Wu, J. Jiang, X. Gu, and C. Tong, "Nitrogen and sulfur codoped carbon quantum dots for highly selective and sensitive fluorescent detection of $\mathrm{Fe}$ (III) ions and L-cysteine," Microchimica Acta, vol. 184, no. 7, pp. 2291-2298, 2017.

[2] Y. Dong, G. Li, N. Zhou, R. Wang, Y. Chi, and G. Chen, "Graphene quantum dot as a green and facile sensor for free chlorine in drinking water," Analytical Chemistry, vol. 84, no. 19, pp. 8378-8382, 2012.

[3] S. Benítez-Martínez, Á. I. López-Lorente, and M. Valcárcel, "Graphene quantum dots sensor for the determination of graphene oxide in environmental water samples," Analytical Chemistry, vol. 86, no. 24, pp. 12279-12284, 2014.

[4] Z. L. Wu, M. X. Gao, T. T. Wang, X. Y. Wan, L. L. Zheng, and C. Z. Huang, "A general quantitative $\mathrm{pH}$ sensor developed with dicyandiamide $\mathrm{N}$-doped high quantum yield graphene quantum dots," Nanoscale, vol. 6, no. 7, pp. 3868-3874, 2014.

[5] X. Zhou, P. Ma, A. Wang et al., "Dopamine fluorescent sensors based on polypyrrole/graphene quantum dots core/shell hybrids," Biosensors \& Bioelectronics, vol. 64, pp. 404-410, 2015.

[6] J. Shi, C. Chan, Y. Pang et al., "A fluorescence resonance energy transfer (FRET) biosensor based on graphene quantum dots (GQDs) and gold nanoparticles (AuNPs) for the detection of mecA gene sequence of Staphylococcus aureus," Biosensors and Bioelectronics, vol. 67, no. 5, pp. 595-600, 2015.

[7] Y. He, J. Sun, D. Feng, H. Chen, F. Gao, and L. Wang, "Graphene quantum dots: highly active bifunctional nanoprobes for nonenzymatic photoluminescence detection of hydroquinone," Biosensors and Bioelectronics, vol. 74, pp. 418-422, 2015.

[8] M. Liu, T. Liu, Y. Li et al., "A FRET chemsensor based on graphene quantum dots for detecting and intracellular imaging of $\mathrm{Hg}^{2+}$," Talanta, vol. 143, no. 9, pp. 442-449, 2015.

[9] S. Umrao, M.-H. Jang, J.-H. Oh et al., "Microwave bottom-up route for size-tunable and switchable photo- luminescent graphene quantum dots using acetylacetone: new platform for enzyme-free detection of hydrogen peroxide," Graphene, vol. 81, no. 1, pp. 514-524, 2015.

[10] L. Wang, Y. Wang, T. Xu et al., "Gram-scale synthesis of single-crystalline graphene quantum dots with superior optical properties," Nature Communications, vol. 5, no. 1, 2014.

[11] M. Xie, Y. Su, X. Lu, Y. Zhang, Z. Yang, and Y. Zhang, "Blue and green photoluminescence graphene quantum dots synthesized from carbon fibers," Materials Letters, vol. 93, no. 1, pp. 161-164, 2013.

[12] Y. He, X. Wang, J. Sun et al., "Fluorescent blood glucose monitor by hemin-functionalized graphene quantum dots based sensing system," Analytica Chimica Acta, vol. 810, pp. 71-78, 2014.

[13] Y. N. Hao, H. L. Guo, L. Tian, and X. Kang, "Enhanced photoluminescence of pyrrolic-nitrogen enriched graphene quantum dots," RSC Advances, vol. 5, no. 54, pp. 4375043755, 2015.

[14] Z. Fan, Y. Li, X. Li et al., "Surrounding media sensitive photoluminescence of boron-doped graphene quantum dots for highly fluorescent dyed crystals, chemical sensing and bioimaging," Carbon, vol. 70, pp. 149-156, 2014.

[15] G. Wang, Q. Guo, D. Chen et al., "Facile and highly effective synthesis of controllable lattice sulfur-doped graphene quantum dots via hydrothermal treatment of durian," ACS Applied Materials \& Interfaces, vol. 10, no. 6, pp. 5750-5759, 2018.

[16] W. Zou, L. Tang, J. Xiang et al., "Functionalization of graphene quantum dots by fluorine: Preparation, properties, application, and their mechanisms," App. Phys. Lett., vol. 110, no. 22, pp. 221901-221905, 2017.

[17] Y. Li, S. Li, Y. Wang et al., "Electrochemical synthesis of phosphorus-doped graphene quantum dots for free radical scavenging," Physical Chemistry Chemical Physics, vol. 19, no. 18, pp. 11631-11638, 2017.

[18] N. Far'ain Md Noor, M. A. Saiful Badri, M. M. Salleh, and A. A. Umar, "Synthesis of white fluorescent pyrrolic nitrogen-doped graphene quantum dots," Optical Materials, vol. 83, pp. 306314, 2018.

[19] X. Kong, Y. Gong, and Z. Fan, "The sensitive turn-on fluorescence detection of ascorbic acid based on iron(III)-modulated nitrogen-doped graphene quantum dots," Journal of Fluorescence, vol. 26, no. 5, pp. 1755-1762, 2016.

[20] S. Dey, A. Govindaraj, K. Biswas, and C. N. R. Rao, "Luminescence properties of boron and nitrogen doped graphene quantum dots prepared from arc-discharge-generated doped graphene samples," Chemical Physics Letters, vol. 595-596, no. 3, pp. 203-208, 2014.

[21] Z. Guo, B. Cai, Q. Cao et al., "Facile synthesis of aminefunctionalized graphene quantum dots with highly $\mathrm{pH}$ sensitive photoluminescence," Fullerenes, Nanotubes, and Carbon Nanostructures, vol. 25, no. 12, pp. 704-709, 2017.

[22] F. Gosetti, V. Gianotti, S. Angioi, S. Polati, E. Marengo, and M. C. Gennaro, "Oxidative degradation of food dye E133 Brilliant Blue FCF: liquid chromatography-electrospray mass spectrometry identification of the degradation pathway," Journal of Chromatography. A, vol. 1054, no. 1-2, pp. 379-387, 2004.

[23] K. S. Minioti, C. F. Sakellariou, and N. S. Thomaidis, "Determination of 13 synthetic food colorants in water-soluble foods by reversed-phase high-performance liquid chromatography coupled with diode-array detector," Analytica Chimica Acta, vol. 583, no. 1, pp. 103-110, 2007.

[24] M. Khanavi, M. Hajimahmoodi, A. M. Ranjbar, M. R. Oveisi, M. R. S. Ardekani, and G. Mogaddam, "Development of a green chromatographic method for simultaneous determination of food colorants," Food Analytical Methods, vol. 5, no. 3, pp. 408-415, 2012.

[25] N. Pourreza and M. Ghomi, "Simultaneous cloud point extraction and spectrophotometric determination of carmoisine and brilliant blue FCF in food samples," Talanta, vol. 84, no. 1, pp. 240-243, 2011.

[26] R. Li, Z. T. Jiang, and R. X. Wang, "Solid phase extraction combined direct spectrophotometric determination of brilliant blue in food using $\beta$-cyclodextrin polymer," Food Anal Methods, vol. 2, no. 4, pp. 264-270, 2009.

[27] X. CHEN, J. YIN, C. ZHANG, N. LU, and Z. CHEN, "Determination of Brilliant Blue FCF by a novel solid-state 
ECL quenching sensor of $\mathrm{Ru}(\mathrm{bpy})_{3}{ }^{2+}$-poly(sulfosalicylic acid)/GCE," Analytical Sciences, vol. 33, no. 10, pp. 11231128, 2017.

[28] M. A. Prado, L. F. V. Boas, M. R. Bronze, and H. T. Godoy, "Validation of methodology for simultaneous determination of synthetic dyes in alcoholic beverages by capillary electrophoresis," Journal of Chromatography A, vol. 1136, no. 2, pp. 231-236, 2006.

[29] S. M. Ghoreishi, M. Behpour, and M. Golestaneh, "Simultaneous voltammetric determination of brilliant blue and tartrazine in real samples at the surface of a multi-walled carbon nanotube paste electrode," Analytical Methods, vol. 3, no. 12, p. 2842, 2011.

[30] R. A. Medeiros, B. C. Lourencao, R. C. Rocha-Filho, and O. Fatibello-Filho, "Simultaneous voltammetric determination of synthetic colorants in food using a cathodically pretreated boron-doped diamond electrode," Talanta, vol. 97, pp. 291-297, 2012.

[31] Y. Dong, J. Shao, C. Chen et al., "Blue luminescent graphene quantum dots and graphene oxide prepared by tuning the carbonization degree of citric acid," Carbon, vol. 50, no. 12, pp. 4738-4743, 2012.

[32] A. H. Gore, M. B. Kale, P. V. Anbhule, S. R. Patil, and G. B. Kolekar, "A novel $\{$ FRET $\}$ probe for selective and sensitive determination of vitamin B12by functionalized $\{\mathrm{CdS}\}$ \{QDs\} in aqueous media: applications to pharmaceutical and biomedical analysis," RSC Advances, vol. 4, no. 2, pp. 683-692, 2014.

[33] J. Zhang, L. Na, Y. Jiang, D. Lou, and L. Jin, "Graphene quantum dots as a fluorescence-quenching probe for quantitative analysis of Ponceau 4R solution," Analytical Methods, vol. 8, no. 39, pp. 7242-7246, 2016. 


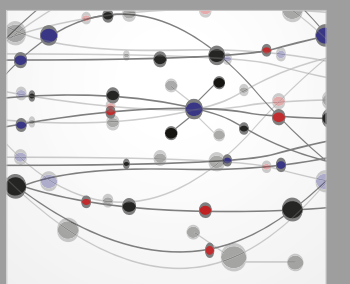

The Scientific World Journal
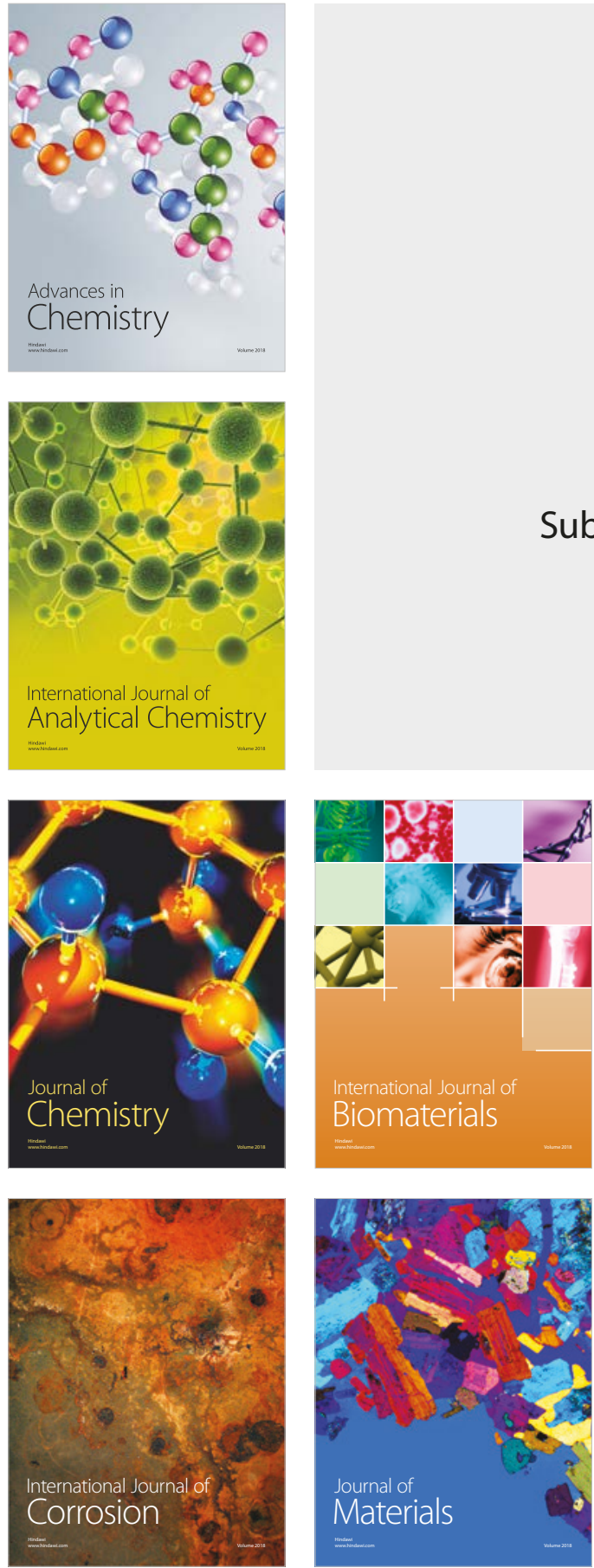

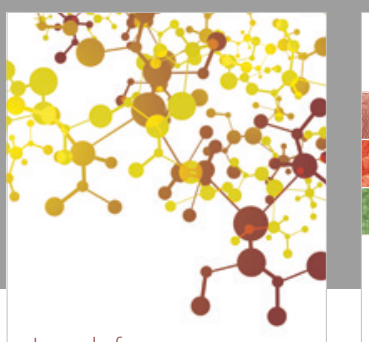

Journal of

Applied Chemistry
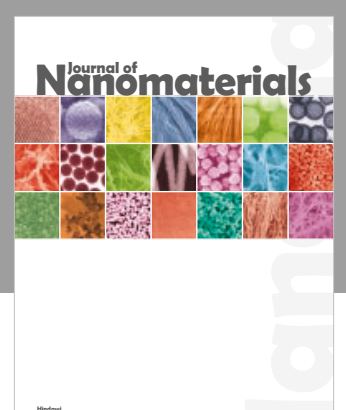

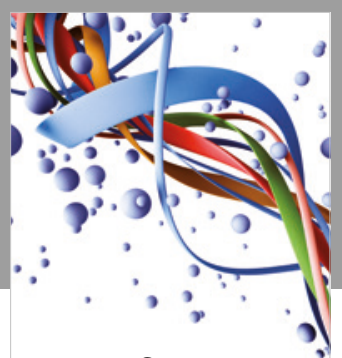

Scientifica

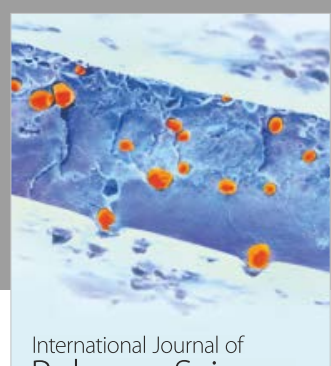

Polymer Science

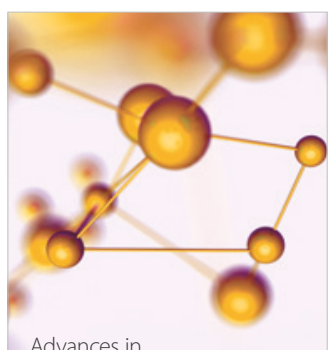

Physical Chemistry
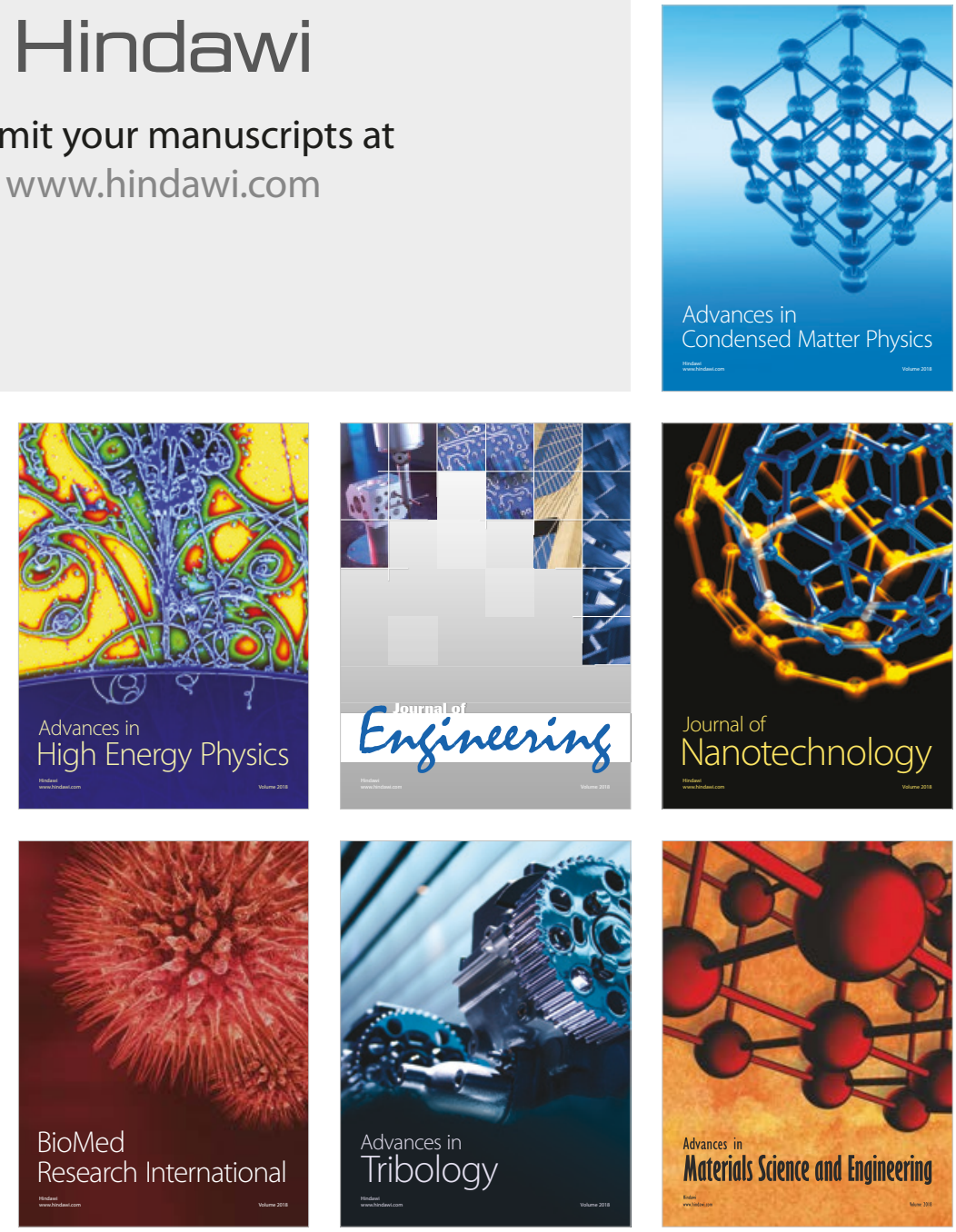